\title{
On minimal distance-regular Cayley graphs of generalized dihedral groups
}

\author{
Štefko Miklavič* \\ University of Primorska, IAM, Muzejski trg 2, 6000 Koper, Slovenia \\ IMFM, Jadranska 19, 1000 Ljubljana, Slovenia \\ stefko.miklavic@upr.si \\ Primož Šparl ${ }^{\dagger}$ \\ University of Ljubljana, Faculty of Education, Kardeljeva ploščad 16, \\ 1000 Ljubljana, Slovenia \\ University of Primorska, IAM, Muzejski trg 2, 6000 Koper, Slovenia \\ IMFM, Jadranska 19, 1000 Ljubljana, Slovenia \\ primoz.sparl@pef.uni-lj.si
}

Submitted: Aug 3, 2020; Accepted: Nov 9, 2020; Published: Nov 27, 2020

(C) Š. Miklavič and P. Šparl. Released under the CC BY-ND license (International 4.0).

\begin{abstract}
Let $G$ denote a finite generalized dihedral group with identity 1 and let $S$ denote an inverse-closed subset of $G \backslash\{1\}$, which generates $G$ and for which there exists $s \in$ $S$, such that $\left\langle S \backslash\left\{s, s^{-1}\right\}\right\rangle \neq G$. In this paper we obtain the complete classification of distance-regular Cayley graphs $\operatorname{Cay}(G ; S)$ for such pairs of $G$ and $S$.
\end{abstract}

Mathematics Subject Classifications: 05E18, 05E30

\section{Introduction}

Two of the most extensively studied phenomena in graph theory are regularity and symmetry properties of graphs. While it is usually quite easy to see that a certain degree of symmetry that a graph possesses implies also certain degree of regularity (for example, every vertex-transitive graph is also regular in a sense that every vertex has the same degree), the opposite question seems to be much harder to handle. It is therefore necessary

\footnotetext{
* Supported in part by the Slovenian Research Agency (research program P1-0285 and research projects N1-0062, J1-9110, J1-1695, N1-0140).

†Supported in part by the Slovenian Research Agency (research program P1-0285 and research projects J1-9108, J1-9110, J1-1694, J1-1695).
} 
to restrict to a particular class of graphs when one studies the relation between the above two concepts.

A connected finite graph is distance-regular if the cardinality of the intersection of two spheres depends only on their radii and the distance between their centres. Even though this condition is purely combinatorial, the notion of distance-regular graphs is closely related to certain topics in algebra, and has motivated a development of various new algebraic notions, as well as shed some new light on the existing ones. Distance-regular graphs are examples of highly regular graphs. However, the problem of determining which of them are Cayley over a group $G$ with respect to a connection set $S$ (see Section 2 for the definition of a Cayley graph) still seems to be difficult to handle. In the existing literature on this topic researchers therefore usually impose certain restrictions either on the diameter of the studied graphs, on their valency, on their eigenvalues, or on the group $G$ and/or the connection set $S$.

Among distance-regular Cayley graphs, those of diameter 2 (also called strongly regular Cayley graphs) have been investigated most thoroughly. Such graphs are equivalent to socalled regular partial difference sets (see [7] for the survey of this topic), and many results on strongly regular Cayley graphs are formulated in the language of partial difference sets. Though many authors investigated such graphs, a complete classification still seems to be beyond reach. In fact, not even strongly regular Cayley graphs of abelian groups have been classified. However, cyclic groups seem to be easier to handle. Strong regularity of circulants (that is Cayley graphs of cyclic groups) has been investigated by several authors and a complete classification of strongly regular circulants was independently achieved by Bridges and Mena [2], Ma [8], and partially by Marušič [9]. More recently Leifman and Muzychuck [6] classified strongly regular Cayley graphs on $\mathbb{Z}_{p^{n}} \times \mathbb{Z}_{p^{n}}, p$ a prime.

While in the references cited in the above paragraph the diameter of the graph was restricted to be 2, van Dam and Jazaeri [5] choose to restrict the valency of a distanceregular graph. They determined all distance-regular Cayley graphs with valency 3 and 4, and they characterized the Cayley graphs among all distance-regular graphs with valency 5 and with one of the known feasible intersection arrays. They also considered distanceregular graphs with girth 3 and valency 6 or 7 .

Regarding the restrictions on the eigenvalues, in [1] the authors classified the distanceregular Cayley graphs with least eigenvalue -2 and diameter at most three.

As for the restrictions on the group $G$, distance-regular Cayley graphs over cyclic and dihedral groups were (almost) classified in $[10,11]$.

In [12], the authors chose to restrict the group $G$ as well as the connection set $S$. We say that the connection set $S$ is minimal, if it generates $G$, but there exists an element $s \in S$ such that $S \backslash\left\{s, s^{-1}\right\}$ does not generate $G$. In [12] the authors obtained a complete classification of distance-regular graphs, which are Cayley over an abelian group and the corresponding connection set $S$ is minimal. In this paper the work from [12] is extended by classifying distance-regular graphs, which are Cayley over a generalized dihedral group, and for which the corresponding connection set $S$ is minimal. Our main result is the following theorem (see Section 2 for the definitions of the graphs appearing 
in the theorem).

Theorem 1. Let $G$ be a generalized dihedral group of order at least 4 with identity 1 and let $S$ be an inverse closed subset of $G \backslash\{1\}$ which generates $G$ and for which there exists $s \in S$ such that $\left\langle S \backslash\left\{s, s^{-1}\right\}\right\rangle \neq G$. Then the Cayley graph Cay $(G ; S)$ is distance-regular if and only if it is isomorphic to one of the following graphs:

(i) The complete bipartite graph minus a 1 -factor $K_{6,6}-6 K_{2}$.

(ii) The Pappus graph.

(iii) The cycle $C_{n}$ for $n \geqslant 4$ even.

(v) The Hamming graph $H(d, n)$, where $d \geqslant 2$ and $n \in\{2,4\}$.

(vi) The Doobs graph $D(n, m)$ where $n, m \geqslant 1$.

(vii) The antipodal quotient of the Hamming graph $H(d, 2)$, where $d \geqslant 4$.

Surprisingly enough, considerably enlarging the set of admissible groups yields only one new distance-regular Cayley graph with respect to a minimal connection set. More precisely, the Pappus graph is the only distance-regular Cayley graph of a generalized dihedral group with respect to a minimal connection set, that is not also a Cayley graph of an abelian group with respect to a minimal connection set.

Our paper is organized as follows. After some preliminaries in Section 2 we first show that every graph from Theorem 1 is indeed a Cayley graph over a generalized dihedral group with respect to a certain minimal connection set in Section 3. In Section 4 we obtain a characterization of distance-regular graphs which are Cartesian products of two non-trivial graphs. In Sections 5 and 6 we finally prove that every Cayley graph over a generalized dihedral group with respect to a certain minimal connection set is contained in the list of Theorem 1.

\section{Preliminaries}

In this section we review some definitions and basic facts about distance-regular graphs and Cayley graphs. More background information on distance-regular graphs can be found in [3].

Throughout this paper all graphs are assumed to be finite, undirected and without loops or multiple edges. For a graph $\Gamma$ we let $V=V(\Gamma), E=E(\Gamma)$ and $\partial_{\Gamma}$ (or just $\partial$ ) denote the vertex set, the edge set and the path length distance function, respectively. The diameter $\max \{\partial(x, y) \mid x, y \in V(\Gamma)\}$ of $\Gamma$ will be denoted by $d_{\Gamma}$ (or just $d$, when the graph $\Gamma$ is clear form the context).

For a vertex $x \in V(\Gamma)$ and an integer $i$ we let $N_{i}^{\Gamma}(x)=\{y \mid \partial(x, y)=i\}$ denote the $i$-th sphere centred at $x$. We abbreviate $N_{i}^{\Gamma}=N_{i}$ when $\Gamma$ is clear from the context. We 
also abbreviate $N(x)=N_{1}(x)$. For a connected graph $\Gamma$ and $x, y \in V$ with $\partial(x, y)=i$ we denote

$c_{i}(x, y)=\left|N_{i-1}(x) \cap N(y)\right|, \quad a_{i}(x, y)=\left|N_{i}(x) \cap N(y)\right|, \quad b_{i}(x, y)=\left|N_{i+1}(x) \cap N(y)\right|$.

If $c_{i}(x, y)\left(a_{i}(x, y), b_{i}(x, y)\right.$ respectively) does not depend on the choice of $x, y$ with $\partial(x, y)=i$ (but only depends on the distance $i$ between $x$ and $y$ ), then we say that the intersection number $c_{i}\left(a_{i}, b_{i}\right.$, respectively) exists for $\Gamma$ and we set $c_{i}=c_{i}(x, y)$ $\left(a_{i}=a_{i}(x, y), b_{i}=b_{i}(x, y)\right)$, where $x, y \in V$ with $\partial(x, y)=i$. Observe that if the intersection numbers $a_{1}, a_{2}, \ldots, a_{i}$ all exist, then $a_{1}=a_{2}=\cdots=a_{i}=0$ holds if and only if for each $j \in\{3,5, \ldots, 2 i+1\}$ there is no cycle of length $j$ in $\Gamma$. Observe also that $a_{0}, c_{0}$, $c_{1}$ and $b_{d}$ always exist and $a_{0}=0, c_{0}=0, c_{1}=1$ and $b_{d}=0$ holds. A connected graph $\Gamma$ with diameter $d$ is said to be distance-regular whenever the intersection numbers $c_{i}, a_{i}$ and $b_{i}$ exist for all $0 \leqslant i \leqslant d$. Note that a distance-regular graph $\Gamma$ is regular with valency $k=b_{0}$, and

$$
a_{i}+b_{i}+c_{i}=k \quad(0 \leqslant i \leqslant d) .
$$

Note also that $b_{i} \neq 0$ for $0 \leqslant i \leqslant d-1$ and $c_{i} \neq 0$ for $1 \leqslant i \leqslant d$. The array

$$
\left\{b_{0}, b_{1}, \ldots, b_{d-1} ; c_{1}, c_{2}, \ldots, c_{d}\right\}
$$

is called the intersection array of $\Gamma$.

For a positive integer $n$ we denote by $K_{n}$ the complete graph on $n$ vertices, and by $C_{n}(n \geqslant 3)$ the cycle on $n$ vertices.

For graphs $\Gamma_{1}$ and $\Gamma_{2}$, their Cartesian product is the graph $\Gamma_{1} \square \Gamma_{2}$ with vertex-set $V\left(\Gamma_{1}\right) \times V\left(\Gamma_{2}\right)$, where vertices $\left(u_{1}, v_{1}\right),\left(u_{2}, v_{2}\right) \in V\left(\Gamma_{1}\right) \times V\left(\Gamma_{2}\right)$ are adjacent if and only if $u_{1}=u_{2}$ and $v_{1}, v_{2}$ are adjacent in $\Gamma_{2}$, or $u_{1}, u_{2}$ are adjacent in $\Gamma_{1}$ and $v_{1}=v_{2}$. It is well known that

$$
\partial_{\Gamma_{1} \square \Gamma_{2}}\left(\left(u_{1}, v_{1}\right),\left(u_{2}, v_{2}\right)\right)=\partial_{\Gamma_{1}}\left(u_{1}, u_{2}\right)+\partial_{\Gamma_{2}}\left(v_{1}, v_{2}\right) .
$$

For positive integers $d$ and $q$, the Hamming graph $H(d, q)$ is the Cartesian product of $d$ copies of the complete graph $K_{q}$. Note that in the case of $q=2$ (these graphs are known as the hypercube graphs) each vertex has a unique counterpart, the so-called antipodal vertex, at maximal distance $d$. Such a pair of antipodal vertices is thus a block of imprimitivity for the automorphism group of $H(d, 2)$. The antipodal quotient of the graph $H(d, 2)$ has as vertices the above mentioned blocks consisting of pairs of antipodal vertices with two such blocks adjacent whenever there is an edge between these blocks in $H(d, 2)$. By [3][Proposition 4.2.2] the antipodal quotient of $H(d, 2)$ is a distance-regular graph. For a nonnegative integer $n$ and a positive integer $m$, the Doobs graph $D(n, m)$ is the Cartesian product of $H(n, 4)$ with $m$ copies of the Shrikhande graph [13] (where in the case of $n=0$ we just take the Cartesian product of $m$ copies of the Shrikhande graph). The Doobs graph $D(n, m)$ is distance-regular with the same intersection numbers as $H(n+2 m, 4)($ see $[3$, page 262]).

For a group $G$ and an element $g \in G$ we denote the order of $g$ by $|g|$. Moreover, for a subgroup $H \leqslant G$ we denote its index in $G$ by $[G: H]$. 
Let $G$ denote a finite group with identity 1 and let $S$ denote an inverse-closed subset of $G \backslash\{1\}$. The Cayley graph $\operatorname{Cay}(G ; S)$ of the group $G$ with respect to the connection set $S$ is the graph with vertex-set $G$, in which $g \in G$ is adjacent with $h \in G$ if and only if $h=g s$ for some $s \in S$. Observe that $\operatorname{Cay}(G ; S)$ is regular with valency $k=|S|$ and is connected if and only if $\langle S\rangle=G$. We say that the connection set $S$ is minimal with respect to $s \in S$ if $G=\langle S\rangle$ but $\left\langle S \backslash\left\{s, s^{-1}\right\}\right\rangle \neq G$. Note also that the left regular representation of $G$ acts as a regular subgroup of the automorphism group of Cay $(G ; S)$. It thus clearly follows that if $H \leqslant G$ then for each $g_{1}, g_{2} \in G$ the subgraphs of Cay $(G ; S)$ induced on the cosets $g_{1} H$ and $g_{2} H$ are isomorphic.

A group $G$ is a generalized dihedral group if it has an index 2 abelian subgroup $A$ and an involution $t \in G \backslash A$ such that tat $=a^{-1}$ holds for all $a \in A$. In other words, $G$ is the semidirect product $A \rtimes\langle t\rangle$, where $A$ is abelian and $t$ is an involution inverting each $a \in A$ by conjugation. In this case, we denote this generalized dihedral group by $G D(A)$. Observe that if $G=G D(A)$ then $G=A \cup t A$. Moreover, the only abelian generalized dihedral groups are the elementary abelian 2-groups. Therefore, $G D(A)$ is not cyclic, unless $A$ is trivial.

\section{The graphs from the main theorem}

In this section we show that the graphs from Theorem 1 are all indeed distance-regular Cayley graphs of generalized dihedral groups with respect to a minimal connection set. That all of them are distance-regular follows from [3]. The following claims can be verified easily.

The graph $K_{6,6}-6 K_{2}$ can be obtained as the Cayley graph of the (generalized) dihedral group $G D(\langle a\rangle)$, where $|a|=6$, with respect to the connection set $S=\left\{a^{ \pm 1}, t, t a^{2}, t a^{4}\right\}$. Note that $S$ is minimal with respect to $a$.

The Pappus graph can be obtained as the Cayley graph of the generalized dihedral group $G D(\langle a\rangle \times\langle b\rangle)$, where $|a|=|b|=3$, with respect to the connection set $S=\{t, t a, t b\}$. The set $S$ is minimal with respect to $t a$.

The cycle $C_{2 n}$, where $n \geqslant 2$, is clearly the Cayley graph of the dihedral group $D_{n}=$ $G D(\langle a\rangle)$, where $|a|=n$, with respect to $S=\{t, t a\}$.

The hypercube $H(d, 2)$, where $d \geqslant 2$, is the Cayley graph of the elementary abelian group $G D\left(\left\langle a_{1}\right\rangle \times \cdots \times\left\langle a_{d-1}\right\rangle\right)$, where $\left|a_{1}\right|=\cdots=\left|a_{d-1}\right|=2$, with respect to $S=$ $\left\{t, a_{1}, \ldots, a_{d-1}\right\}$.

The Hamming graph $H(d, 4)$, where $d \geqslant 2$, is the Cayley graph of the generalized dihedral group $G=G D\left(\langle a\rangle \times\left\langle b_{1}\right\rangle \times \cdots \times\left\langle b_{d-1}\right\rangle\right)$, where $|a|=2$ and $\left|b_{i}\right|=4$ for all $1 \leqslant$ $i \leqslant d-1$, with respect to the connection set $S=\left\{t, a, t a, b_{1}^{ \pm 1}, b_{1}^{2}, b_{2}^{ \pm 1}, b_{2}^{2}, \ldots, b_{d-1}^{ \pm 1}, b_{d-1}^{2}\right\}$. To see this, first note that each element of $G$ can be uniquely represented as $b_{1}^{i_{1}} \cdots b_{d-1}^{i_{d-1}} a^{j} t^{\ell}$, where $i_{1}, i_{2}, \ldots, i_{d-1} \in \mathbb{Z}_{4}$ and $j, \ell \in \mathbb{Z}_{2}$. A corresponding isomorphism can thus be obtained by taking each $b_{1}^{i_{1}} \cdots b_{d-1}^{i_{d-1}} a^{j} t^{\ell}$ to the $d$-tuple $\left(i_{1}, i_{2}, \ldots, i_{d-1}, i_{d}\right)$, where $i_{d}=$ $0,1,2,3$, respectively, if the pair $(j, \ell)$ is $(0,0),(0,1),(1,1),(1,0)$, respectively. The set $S$ is minimal with respect to $b_{i}$ for any $1 \leqslant i \leqslant d-1$. 
The antipodal quotient of the hypercube $H(d, 2)$, where $d \geqslant 4$, can be realised as the graph with vertex set $\mathbb{Z}_{2}^{d-1}$, in which two $(d-1)$-tuples are adjacent whenever they differ in exactly one position or in all positions. Therefore, this graph is the Cayley graph of the generalized dihedral group $G=G D\left(\langle a\rangle \times\left\langle b_{1}\right\rangle \times \cdots \times\left\langle b_{d-4}\right\rangle\right)$, where $|a|=4$ and $\left|b_{i}\right|=2$ for all $1 \leqslant i \leqslant d-4$, with respect to the connection set $S=\left\{a^{ \pm 1}, b_{1}, b_{2}, \ldots, b_{d-4}, t, t a^{2} b_{1} b_{2} \cdots b_{d-4}\right\}$. To see this, first note that each element of $G$ can be uniquely represented as $b_{1}^{i_{1}} \cdots b_{d-1}^{i_{d-1}} a^{j} t^{\ell}$, where $i_{1}, i_{2}, \ldots, i_{d-4}, \ell \in \mathbb{Z}_{2}$ and $j \in \mathbb{Z}_{4}$. A corresponding isomorphism can thus be obtained by taking each $b_{1}^{i_{1}} \cdots b_{d-4}^{i_{d-4}} a^{j} t^{\ell}$ to the $(d-1)$-tuple $\left(i_{1}, i_{2}, \ldots, i_{d-4}, j_{1}, j_{2}, \ell\right)$, where the pair $\left(j_{1}, j_{2}\right)$ equal $(0,0),(0,1),(1,1),(1,0)$, respectively, whenever $j$ is $0,1,2,3$, respectively. The set $S$ is minimal with respect to $a$.

It can be verified that the Shrikhande graph is isomorphic to the Cayley graph of the generalized dihedral group $G D(\langle a\rangle \times\langle b\rangle)$, where $|a|=2$ and $|b|=4$, with respect to $S=\left\{t, t a, t b, t a b^{3}, b^{ \pm 1}\right\}$. But this graph is also isomorphic to the Cayle graph of the abelian group $\langle c\rangle \times\langle d\rangle$, where $|c|=|d|=4$, with respect to the connection set $S=\left\{c^{ \pm 1}, d^{ \pm 1},(c d)^{ \pm 1}\right\}$. Therefore, the Doobs graph $D(n, m)$, where $n, m \geqslant 1$, can be obtained as follows. Let $G$ be the generalized dihedral group

$$
G D\left(\langle a\rangle \times\langle b\rangle \times\left\langle c_{1}\right\rangle \times \cdots \times\left\langle c_{m-1}\right\rangle \times\left\langle d_{1}\right\rangle \times \cdots \times\left\langle d_{m-1}\right\rangle \times\left\langle e_{1}\right\rangle \times \cdots \times\left\langle e_{n}\right\rangle\right),
$$

where $|a|=2$ and $|b|=\left|c_{i}\right|=\left|d_{i}\right|=\left|e_{j}\right|=4$ for all $1 \leqslant i \leqslant m-1$ and $1 \leqslant j \leqslant n$. Furthermore, let $S=S_{0} \cup S_{1} \cup S_{2}$, where $S_{0}=\left\{t, t a, t b, t a b^{3}, b^{ \pm 1}\right\}$,

$$
S_{1}=\left\{c_{1}^{ \pm 1}, \ldots, c_{m-1}^{ \pm 1}, d_{1}^{ \pm 1}, \ldots, d_{m-1}^{ \pm 1},\left(c_{1} d_{1}\right)^{ \pm 1}, \ldots,\left(c_{m-1} d_{m-1}\right)^{ \pm 1}\right\},
$$

and $S_{2}=\left\{e_{1}^{ \pm 1}, e_{1}^{2}, \ldots, e_{n}^{ \pm 1}, e_{n}^{2}\right\}$. A corresponding isomorphism can now be obtained similarly as in the above two cases. Moreover, the set $S$ is minimal with respect to any $e_{i}$, $1 \leqslant i \leqslant n$.

\section{Distance-regular Cartesian products}

In this short section we give a characterization of distance-regular Cartesian products of graphs.

Proposition 2. Let $\Gamma_{1}$ and $\Gamma_{2}$ be nontrivial graphs with diameter $d_{1}$ and $d_{2}$, respectively. Let $\Gamma=\Gamma_{1} \square \Gamma_{2}$. Then $\Gamma$ is distance-regular if and only if $\Gamma_{1}$ is distance-regular with the same intersection numbers as $H\left(d_{1}, q\right)$ and $\Gamma_{2}$ is distance-regular with the same intersection numbers as $H\left(d_{2}, q\right)$ for some $q \geqslant 2$.

Proof. If $\Gamma_{1}$ is distance-regular with the same intersection numbers as $H\left(d_{1}, q\right)$ and $\Gamma_{2}$ is distance-regular with the same intersection numbers as $H\left(d_{2}, q\right)$, then the result follows from [14]. Assume now that $\Gamma$ is distance-regular. We will prove that $\Gamma_{1}$ is distanceregular. The proof that $\Gamma_{2}$ is distance-regular is analogous. By (3) the diameter of $\Gamma$ is $d_{1}+d_{2}$. Let $a_{i}, b_{i}, c_{i}\left(0 \leqslant i \leqslant d_{1}+d_{2}\right)$ be the intersection numbers of $\Gamma$. Pick a vertex $w \in V\left(\Gamma_{2}\right)$ and note that a subgraph of $\Gamma$, induced on $\left\{(x, y) \mid x \in V\left(\Gamma_{1}\right)\right\}$ is isomorphic 
to $\Gamma_{1}$. As $\Gamma$ is regular, so is $\Gamma_{1}$. Pick vertices $x, y$ of $\Gamma_{1}$ and let $i=\partial_{\Gamma_{1}}(x, y)$. Note that $\partial_{\Gamma}((x, w),(y, w))$ is also equal to $i$. Let $C_{i}(x, y)=N_{i-1}^{\Gamma_{1}}(x) \cap N^{\Gamma_{1}}(y), A_{i}(x, y)=$ $N_{i}^{\Gamma_{1}}(x) \cap N^{\Gamma_{1}}(y), B_{i}(x, y)=N_{i+1}^{\Gamma_{1}}(x) \cap N^{\Gamma_{1}}(y)$. It follows from the definition of Cartesian product that $N_{i-1}^{\Gamma}((x, w)) \cap N^{\Gamma}((y, w))=C_{i}(x, y) \times\{w\}$ and $N_{i}^{\Gamma}((x, w)) \cap N^{\Gamma}((y, w))=$ $A_{i}(x, y) \times\{w\}$. As $\Gamma$ is distance-regular, we have that $\left|C_{i}(x, y)\right|=c_{i}$ and $\left|A_{i}(x, y)\right|=a_{i}$. Since $\Gamma_{1}$ is regular (say of valency $k_{1}$ ), we also have that $\left|B_{i}(x, y)\right|=k_{1}-c_{i}-a_{i}$. It follows that $\Gamma_{1}$ is distance-regular. The result now follows from [14].

If $q \neq 4$, then the Hamming graph $H(d, q)$ is uniquely characterised by its intersection numbers. If however $\Gamma$ has the same intersection array as the Hamming graph $H(d, 4)$, then either $\Gamma$ is isomorphic to $H(d, 4)$ or to the Doobs graph $D(d-2 m, m)$ for some $m \geqslant 1$ (see [3, p. 262] for details). We therefore have the following corollary of Proposition 2.

Corollary 3. Let $\Gamma=\Gamma_{1} \square \Gamma_{2}$, where $\Gamma_{1}$ and $\Gamma_{2}$ are nontrivial graphs. If $\Gamma$ is distanceregular then $\Gamma$ is either isomorphic to a Hamming graph $H(d, q)$ or to a Doobs graph $D(n, m)$.

\section{The case $s \in A$}

In this and the next section we complete the proof of Theorem 1. Throughout these two sections we will thus be working with a Cayley graph $\Gamma=\operatorname{Cay}(G ; S)$ of a generalized dihedral group $G=G D(A)$ for some abelian group $A$ of order at least 2 with respect to a connection set $S$, such that $S$ is minimal with respect to some $s \in S$. We distinguish two main cases depending on whether $s \in A$ or not. In this section we focus on the former case.

Note that if $S=\left\{s, s^{\prime}\right\}$, where $s, s^{\prime}$ are involutions, then $\Gamma$ is isomorphic to the cycle of length $\left|s^{\prime} s^{-1}\right|=|G|$. For the rest of this section we will thus restrict ourselves to the case that $|S| \geqslant 3$. For ease of reference we also fix the following notation.

Notation 4. Let $G=G D(A)$ denote a finite generalized dihedral group with identity 1 , where $A$ is a nontrivial abelian group. Let $S$ denote an inverse-closed subset of $G \backslash\{1\}$ with $|S| \geqslant 3$, which generates $G$ and for which there exists $s \in A$, such that $H=\left\langle S \backslash\left\{s, s^{-1}\right\}\right\rangle$ is a proper subgroup of $G$. Assume that $\Gamma=\operatorname{Cay}(G ; S)$ is distance-regular with diameter $d$ and with intersection numbers $a_{i}, b_{i}, c_{i}(0 \leqslant i \leqslant d)$. Let $\Gamma^{\prime}=\operatorname{Cay}\left(H ; S \backslash\left\{s, s^{-1}\right\}\right)$.

With reference to Notation 4 , note that $G$ is the disjoint union of the cosets $s^{i} H$, where $0 \leqslant i \leqslant[G: H]-1$, and that $|s|$ is a multiple of $[G: H]$. Recall also that the subgraphs of $\Gamma$, induced on $s^{i} H(0 \leqslant i \leqslant[G: H]-1)$ are isomorphic to $\Gamma^{\prime}$, and that these subgraphs are connected.

Proposition 5. With reference to Notation 4, the following (i)-(iii) hold:

(i) For every $h \in H$ and for every $0 \leqslant i \leqslant[G: H]-1$, the only neighbours of $s^{i} h$ outside $s^{i} H$ are $s^{i-1} h \in s^{i-1} H$ and $s^{i+1} h \in s^{i+1} H$.

(ii) For every $h \in H$ and for every $0 \leqslant i \leqslant[G: H]-1$, the vertex $s^{i} h$ has two neighbours in $s^{i+1} H$ if and only if $[G: H]=2$ and $|s| \geqslant 4$. 
(iii) The intersection number $c_{2}$ satisfies $c_{2} \geqslant 2$.

Proof. (i) Let $\varepsilon \in\{-1,1\}$. If $h \in A$, then we have

$$
s^{i} h s^{\varepsilon}=s^{i} s^{\varepsilon} h=s^{i+\varepsilon} h \in s^{i+\varepsilon} H .
$$

If however $h=t a$ for some $a \in A$, then

$$
s^{i} h s^{-\varepsilon}=s^{i} \operatorname{tas}^{-\varepsilon}=s^{i} t s^{-\varepsilon} a=s^{i} s^{\varepsilon} t a=s^{i+\varepsilon} h \in s^{i+\varepsilon} H .
$$

Therefore, $s^{i} h$ is adjacent with both $s^{i-1} h$ and $s^{i+1} h$. If however, $s^{\prime} \in S \backslash\left\{s, s^{-1}\right\}$, then $s^{\prime} \in H$, and so clearly $s^{i} h s^{\prime} \in s^{i} H$.

(ii) This is a straightforward corollary of (i) above, since if $[G: H]>2$, then $s^{i-1} H \neq$ $s^{i+1} H$.

(iii) Since $G$ is not cyclic, there exists $h \in S \backslash\langle s\rangle$. Consider the vertices 1 and $s h$. By item (i) above the vertices 1 and $s h \in s H$ are not adjacent (since $h \neq s^{-2}$ ). Moreover, as $s$ and $h$ are clearly their common neighbours, $s \neq h$ implies that $c_{2} \geqslant 2$.

Proposition 6. With reference to Notation \& we have $[G: H] \in\{2,4\}$.

Proof. By way of contradiction, assume $[G: H] \notin\{2,4\}$. Suppose first that $[G: H] \geqslant 5$ and consider the vertices 1 and $s^{2}$ of $\Gamma$. By Proposition 5 the vertices 1 and $s^{2}$ are not adjacent and $s$ is their unique neighbour. This contradicts Proposition 5(iii).

Suppose now that $[G: H]=3$. Assume that $|s| \geqslant 6$. We will first show that in this case we have $|s|=6$. Consider the vertices 1 and $s^{2}$ and note that these vertices are at distance 2 and that $s$ is their common neighbour. By Proposition 5, the vertices 1 and $s^{2}$ must have at least one more common neighbour and the only possibilities for them are $s^{3}$ and $s^{-1}$. In both cases we get that $\left\{s^{3}, s^{-3}\right\} \subseteq S$, and so $s^{3}$ and $s^{-1}$ are both common neighbours of 1 and $s^{2}$, implying that $c_{2}=3$. Consider now the vertices 1 and $s^{4}$. By Proposition 5 they are not adjacent, and so $s^{3} \in S$ implies that they are at distance 2 . Besides their common neighbours $s$ and $s^{3}$ they must thus have an additional common neighbour, which can only be $s^{-1}=s^{5}$. In particular, $s^{6}=1$, implying that $|s|=6$.

As $G$ is generated by $S$, there exists $a \in A$ such that $t a \in S$. Consider now the vertices 1 and $t_{a s}{ }^{-1}=s t a$ and observe that $\partial(1, s t a)=2$ and that $s, t a$ are their common neighbours. As $c_{2}=3$, the vertices 1 and sta must have an additional common neighbour, which by Proposition 5 must be $s^{-1}=s t a s^{-1}$. But then $s t a=1$, a contradiction.

Therefore $|s|=3$ and now a similar argument as in the proof of Proposition 7 shows that $\Gamma \cong K_{3} \square \Gamma^{\prime}$. As a Cartesian product of graphs can be factorized uniquely as a product of prime factors, Corollary 3 implies that $\Gamma$ is isomorphic to the Hamming graph $H(d, 3)$. However, as $\Gamma$ is of even order, this is not possible.

Proposition 7. With reference to Notation 4 assume that $[G: H]=4$. Then $|s|=4$ and $\Gamma$ is isomorphic to the Hamming graph $H(d, 2)$. 
Proof. Suppose first that $|s| \geqslant 5$. Similarly as in the first paragraph of the proof of Proposition 6 we find that $s$ is the unique common neighbour of 1 and $s^{2}$, contradicting Proposition 5(iii). Therefore $|s|=4$. We claim that $\Gamma=\Gamma^{\prime} \square C_{4}$. Let $s^{i} h$, where $h \in H$, be a vertex of $\Gamma$. By Proposition 5 this vertex has precisely two neighbours outside $s^{i} H$, namely $s^{i-1} h \in s^{i-1} H$ and $s^{i+1} h \in s^{i+1} H$. Moreover, any neighbour of $s^{i} h$ from $s^{i} H$ is of the form $s^{i} h s^{\prime}$ for some $s^{\prime} \in S \backslash\left\{s, s^{-1}\right\}$. Since $h s^{\prime} \in H$, Proposition 5 implies that the two neighbours of $s^{i} h s^{\prime}$ outside $s^{i} H$ are $s^{i+1} h s^{\prime}$ and $s^{i-1} h s^{\prime}$. These two vertices are clearly adjacent to $s^{i+1} h$ and $s^{i-1} h$, respectively. Therefore, $\Gamma \cong \Gamma^{\prime} \square C_{4}$, as claimed.

By Corollary 3, $\Gamma$ is either isomorphic to a Hamming graph $H(d, q)$ for some $q$, or to a Doobs graph $D(n, m)$ for some $m, n$. However, note that it follows from Proposition 5 that 1 and $s$ are adjacent vertices with no common neighbours, which forces $a_{1}=0$. Consequently, $\Gamma$ is isomorphic to a Hamming graph $H(d, 2)$.

With reference to Notation 4 , in the last part of this section we study the case $[G$ : $H]=2$. We first consider the case $|s|=2$.

Proposition 8. With reference to Notation 4 assume that $[G: H]=|s|=2$. Then $\Gamma$ is isomorphic to the Hamming graph $H(d, 2)$.

Proof. By Proposition 5 we have that $\Gamma \cong K_{2} \square \Gamma^{\prime}$. As a Cartesian product of graphs can be factorized uniquely as a product of prime factors, it follows from Corollary 3 that $\Gamma$ is isomorphic to the Hamming graph $H(d, 2)$.

We now consider the case $[G: H]=2$ and $|s| \geqslant 4$.

Proposition 9. With reference to Notation 4 assume that $[G: H]=2$ and $|s| \geqslant 4$. Then the following (i), (ii) hold.

(i) The intersection number $a_{1} \in\{0,2\}$. Moreover, $a_{1}=2$ if and only if $s^{2} \in S$.

(ii) The intersection number $c_{2} \in\{2,4\}$. Moreover, if $|s| \geqslant 6$ then $c_{2}=4$.

Proof. (i) Consider adjacent vertices 1 and $s$. By Proposition 5 the only possible common neighbours of these vertices are $s^{2}$ and $s^{-1}$. However, $s^{2}$ is a common neighbour of 1 and $s$ if and only if $s^{2} \in S$ which occurs if and only if $s^{-2} \in S$. But it is easy to see that this occurs if and only if $s^{-1}$ is a common neighbour of 1 and $s$. As $|s| \geqslant 4$ we have that $s^{2} \neq s^{-1}$, and the result follows.

(ii) Pick $a \in A$ such that $t a \in S$ and consider the vertices 1 and $t a s^{-1}=s t a$. Observe that $\partial(1, s t a)=2$ and that $t a$ and $s$ are their common neighbours. By Proposition 5 the only other possible common neighbours of these vertices are $s^{-1}$ and $s^{2} t a$. However, $s^{-1}$ is a common neighbour of 1 and sta if and only if $s^{2} t a \in S$ which occurs if and only if $s^{2} t a$ is a common neighbour of 1 and $s t a$. As $s^{-1} \neq s^{2} t a$, we have that $c_{2} \in\{2,4\}$. To complete the proof assume $|s| \geqslant 6$ and that $c_{2}=2$. As $a_{1} \in\{0,2\}$ (with $a_{1}=2$ if and only if $s^{2} \in S$ ), the vertices 1 and $s^{2}$ must have exactly two common neighbours. As $|s| \geqslant 6$, Proposition 5 implies that $s$ is the unique common neighbour of 1 and $s^{2}$, which is contained in $s H$. Therefore, there exists $h \in H$, which is adjacent to both 1 and $s^{2}$, and so $\left\{s, s^{-1}, h, h^{-1}, s^{-2} h, h^{-1} s^{2}\right\} \subseteq S$. However, as $h \neq s^{2}$, we have that $\partial\left(1, s^{-1} h\right)=2$, 
but $h, s^{-1}$ and $s=s^{-1} h h^{-1} s^{2}$ are three distinct common neighbours of these two vertices, contradicting $c_{2}=2$.

Proposition 10. With reference to Notation 4 assume that $[G: H]=2$ and $|s| \geqslant 6$. Then $\Gamma$ is isomorphic to $K_{6,6}-6 K_{6}$, the complete bipartite graph $K_{6,6}$ minus a 1 -factor.

Proof. Recall that by Proposition 9 we have that $a_{1} \in\{0,2\}$ and $c_{2}=4$. Assume first that $a_{1}=2$, that is, assume that $s^{2} \in S$. We first claim that $\left\{s^{2}, s^{4}, \ldots, s^{|s|-2}\right\} \subseteq S$. Indeed, assume that $\left\{s^{2}, s^{4}, \ldots, s^{2 i}\right\} \subseteq S$ for some $1 \leqslant i<(|s|-2) / 2$. Since $s^{2 i+1} \neq s^{-1}$, Proposition 5 implies that $\partial\left(1, s^{2 i+1}\right)=2$, and so the vertices 1 and $s^{2 i+1}$ must have 4 common neighbours. Again by Proposition 5 these common neighbours are $s, s^{-1}, s^{2 i}$ and $s^{2 i+2}$, which shows that $s^{2 i+2} \in S$. This proves the claim. It follows that $s, s^{4}, s^{6}, \ldots, s^{|s|-2}$ are common neighbours of 1 and $s^{2}$, and so $a_{1}=2$ forces $|s|=6$.

Pick now $a \in A$ such that $t a \in S$ and note that $\partial(1, s t a)=2$. Therefore 1 and sta must have 4 common neighbours, and these common neighbours are $s, s^{-1}, t a$ and $s^{2} t a$, forcing $s^{2} t a \in S$. But now $s, s^{4}$ and $s^{2} t a$ are common neighbours of 1 and $s^{2}$, contradicting $a_{1}=2$.

Therefore, $a_{2}=0$. Pick $h \in S \backslash\langle s\rangle$ and note that $\partial(1, s h)=2$. A similar argument as in the first paragraph of this proof shows that $\left\{h, s^{2} h, s^{4} h, \ldots, s^{|s|-2} h\right\} \subseteq S$. This implies that $s, h, s^{2} h, s^{4} h, \ldots, s^{|s|-2} h$ are all common neighbours of 1 and $s^{2}$, and so $c_{2}=4$ forces that $|s|=6$. Since $s, h, s^{2} h, s^{4} h$ are four distinct common neighbours of 1 and $s^{2}$ the above argument in fact shows that $S \backslash\langle s\rangle=\left\{h, s^{2} h, s^{4} h\right\}$. Since $s^{2}, s^{3}, s^{4} \notin S$ (as $a_{1}=0$ and since $S$ is minimal with respect to $s$ ) this in fact implies that $S=\left\{s, s^{-1}, h, s^{2} h, s^{4} h\right\}$. Since $\Gamma$ is connected, it now follows that $|G|=12$ and that $\Gamma \cong K_{6,6}-6 K_{6}$, as claimed.

This leaves us with the case $[G: H]=2$ and $|s|=4$. The arguments in this case are much longer and technical. Nevertheless, it turns out that one can simply repeat the corresponding proof from [12] in the same situation in the case of distance-regular Cayley graphs of abelian groups (Subsections 7.1 and 7.2). All the arguments go through in our setting. The only thing one needs to change is that whenever in [12] we have a vertex of the form $x s^{i}$ one has to write it as $s^{i} x$ (which, as the group is abelian in that case, changes nothing). In this way we get the following result.

Proposition 11. With reference to Notation 4 assume that $[G: H]=2$ and $|s|=4$. Then $\Gamma$ is isomorphic to the antipodal quotient of the Hamming graph $H(2 d, 2)$ or the antipodal quotient of the Hamming graph $H(2 d+1,2)$, to the Hamming graph $H(d, 4)$ or to the Doobs graph $D(n, m)$ with $n, m \geqslant 1$ and $d=n+2 m$.

\section{$6 \quad$ The case $s \in t A$}

In this section we classify the distance-regular Cayley graphs of generalized dihedral groups $G D(A)$ with respect to some minimal connection set $S$ with respect to some $s \in t A \cap S$. Note that all elements of $t A$ are involutions that invert each element of $A$ by conjugation, so that we can assume $s=t$. Observe that in the case that $|S|=2$ the corresponding graph is a cycle of even length. For the rest of this section we will 
thus be considering examples with $|S| \geqslant 3$. Note that this also implies that $|A| \geqslant 3$, as otherwise $|S| \geqslant 3$ contradicts the assumption that $S$ is minimal with respect to $t$. For ease of reference we adopt the following notation and assumptions.

Notation 12. Let $G=G D(A)$ denote a finite generalized dihedral group with identity 1 , where $A$ is an abelian group of order at least 3. Let $S$ denote an inverse-closed subset of $G \backslash\{1\}$ with $|S| \geqslant 3$, which generates $G$ and such that $H=\langle S \backslash\{t\}\rangle \neq G$. Assume that $\Gamma=\operatorname{Cay}(G ; S)$ is distance-regular with diameter $d$. We define $S_{1}=S \cap A, S_{2}=\{a \in A \mid$ $\left.t a \in S, t a^{-1} \in S\right\}$ and $S_{3}=\left\{a \in A \mid t a \in S, t a^{-1} \notin S\right\}$.

Lemma 13. With reference to Notation 12 each vertex from $H$ has a unique neighbour outside H. Moreover, the following (i), (ii) hold:

(i) For every $a \in H \cap A$ the unique neighbour of a outside $H$ is $t a^{-1} \in t H$.

(ii) For every $t b \in H \backslash A$ the unique neighbour of $t b$ outside $H$ is $b^{-1} \in b^{-1} H \cap H t$. Moreover, for any $t b, t c \in H \backslash A$ the unique neighbour of tc outside $H$ is contained in $b^{-1} H$.

Proof. Since $H=\langle S \backslash\{t\}\rangle$, it is clear that each vertex $x$ from $H$ has a unique neighbour outside $H$ and this neighbour is $x t$. Now, if $x \in A$ then $x t=t x^{-1}$, proving (i). If however, $x=t b$ for some $b \in A$ (recall that $[G: A]=2$ ) then $x t=t b t=b^{-1} \in b^{-1} H$. On the other hand, since $x \in H$ we also have that $x t \in H t$. Finally, if $t b, t c \in H \backslash A$, then $c^{-1} b \in H \cap A$, and so $c^{-1}=b^{-1}\left(c^{-1} b\right)$, implying that $t c t=c^{-1} \in b^{-1} H$.

Proposition 14. With reference to Notation 12, we have $c_{2} \in\{1,2\}$. Moreover, the following (i), (ii) hold.

(i) If $c_{2}=1$, then $S_{1}=\varnothing$ and $S_{2}=\{1\}$. In particular, $\Gamma$ is bipartite.

(ii) If $c_{2}=2$, then $S_{3}=\varnothing$ and $[G: H]=2$.

Proof. Pick $s \in S \backslash\{t\}$. Since $s \in H$, Lemma 13 implies that $\partial(t, s)=2$ and that $s t$ and 1 are the only potential common neighbours of $s$ and $t$. Therefore, $1 \leqslant c_{2} \leqslant 2$, as claimed. Moreover, $c_{2}=1$ if and only if st is not a neighbour of $t$ for each $s \in S \backslash\{t\}$, while $c_{2}=2$ if and only if $s t$ is a neighbour of $t$ for each $s \in S \backslash\{t\}$.

Suppose first that $c_{2}=1$. Since for each $s \in S_{1}$ we have $s^{-1} \in S$ and $s t=t s^{-1}$, the above paragraph implies that $S_{1}=\varnothing$. This clearly implies that $S \subseteq t A$, and so $\Gamma$ is bipartite. Moreover, as for each $a \in S_{2} \backslash\{1\}$ we have that $t a, t a^{-1} \in S$, it follows that $t t a^{-1}=a^{-1}=$ tat is a neighbour of $t$, contradicting $c_{2} \neq 2$. Thus $S_{2}=\{1\}$, as claimed.

Suppose now that $c_{2}=2$ and let $a \in A$ be such that $t a \in S$. By the first paragraph of this proof $a^{-1}=t a t$ is adjacent to $t$, implying that $t a^{-1} \in S$, and so $a \in S_{2}$. Consequently, $S_{3}=\varnothing$, as claimed. To prove that $[G: H]=2$, note first that by Lemma 13 at $\in t H$ for each $a \in H \cap A$. Next, fix $b \in S_{2}$ (note that as $\Gamma$ is connected, we have that $S_{2} \neq \varnothing$ ), and observe that by the above argument we have that $t b t=b^{-1} \in t H$. Now Lemma 13(ii) implies that for every $t c \in H \backslash A$, the unique neighbour of $t c$ outside $H$ is in $t H$. This shows that $H t \subseteq t H$, and so $H t=t H$. But then the edges corresponding to multiplication 
by $t$ induce a perfect matching between $H$ and $t H$, and so connectedness of $\Gamma$ implies that $G=V(\Gamma)=H \cup t H$, forcing $[G: H]=2$.

Proposition 15. With reference to Notation 12, assume that $c_{2}=2$. Then $\Gamma$ is isomorphic to the Hamming graph $H(d, 2)$.

Proof. We claim that $\Gamma \cong \Gamma^{\prime} \square K_{2}$, where $\Gamma^{\prime}$ is the subgraph of $\Gamma$ induced on $H$. By Proposition 14 and its proof we have that $G=H \cup t H$ and that the edges corresponding to multiplication by $t$ induce a perfect matching between $H$ and $t H$. As the left regular representation of $H$ is a subgroup of automorphisms of $\Gamma$ it thus suffices to show that for each $s \in S \backslash\{t\}$ we have that $t$ and st are adjacent. If $s \in A$ then $s^{-1} \in S$, and so $s t=t s^{-1}$ is a neighbour of $t$. If however, $s \notin A$ then by Proposition 14 we have that $s=t a$ for some $a \in A$ with $t a^{-1} \in S$. Thus $s t=t a t=a^{-1}=t t a^{-1}$ is also a neighbour of $t$. This proves our claim.

To complete the proof, note that Corollary 3 and uniqueness of decomposition of Cartesian products into prime factors implies that $\Gamma \cong H(d, 2)$.

Proposition 16. With reference to Notation 12, assume that $c_{2}=1$. Then the following (i)-(iii) hold.

(i) $c_{3} \geqslant 2$.

(ii) If $[G: H] \geqslant 3$, then $c_{3}=2$.

(iii) If $[G: H]=2$, then for every $a, b \in S_{3}, a \neq b$ we have

$$
\left|\left\{\left(c, c^{\prime}\right) \in S_{3} \times S_{3} \mid c c^{\prime}=a^{-1} b\right\}\right|=c_{3}-2 .
$$

Proof. Pick $a, b \in S_{3}, a \neq b$ and consider tatb $=a^{-1} b$. By Proposition 14 the graph $\Gamma$ is bipartite, and so Lemma 13 implies that $\partial\left(t, a^{-1} b\right)=3$.

(i) Since $t t b t a=t b^{-1} a=t a b^{-1}=a^{-1} b t$, we find that $t a \in H$ and $t a b^{-1} \in t H$ are two neighbours of $a^{-1} b$ which are both at distance 2 from $t$. This shows that $c_{3} \geqslant 2$.

(ii) Let us consider all the paths of length 3 from $a^{-1} b$ to $t$. As $c_{2}=1$, there are $c_{3}$ of such paths and each neighbour of $a^{-1} b$ is contained in at most one such path. In particular, only one such path has its second vertex in $t H$ (namely $\left.P_{1}=\left(a^{-1} b, t a b^{-1}, b, t\right)\right)$. Note that $P_{2}=\left(a^{-1} b, t a, 1, t\right)$ is also a path from $a^{-1} b$ to $t$. Therefore, if $c_{3}>2$ then there must also exist a path starting with $\left(a^{-1} b, t a b^{-1} c\right)$ for some $c \in S_{3} \backslash\{b\}$. If the third vertex of this path was in $H$, then by Lemma 13 it would have to be 1 , contradicting $c_{2}=1$ as $t a$ and $t a b^{-1} c$ would be common neighbours of 1 and $a^{-1} b$. Therefore, the third vertex is $t a b^{-1} c t=a^{-1} b c^{-1}$. As this vertex is a neighbour of $t$ and is not 1 , Lemma 13 implies that it is contained in $t H$. But then Lemma 13(ii) implies that $H t \subseteq t H$, contradicting $[G: H] \geqslant 3$.

(iii) The above argument shows that, besides the two paths $P_{1}$ and $P_{2}$, each path of length 3 from $a^{-1} b$ to $t$ is of the form $\left(a^{-1} b, t a b^{-1} c, a^{-1} b c^{-1}, t a b^{-1} c c^{\prime}\right)$, where $c, c^{\prime} \in S_{3}$. Since $t a b^{-1} c c^{\prime}=t$ it follows that the number of paths of length 3 from $a^{-1} b$ to $t$, different from $P_{1}$ and $P_{2}$, equals the number of pairs $\left(c, c^{\prime}\right) \in S_{3} \times S_{3}$, for which $c c^{\prime}=a^{-1} b$. 
Proposition 17. With reference to Notation 12, assume that $c_{2}=1$. Then $[G: H]=3$.

Proof. By Proposition 14 the graph $\Gamma$ is bipartite. Suppose to the contrary that $[G$ : $H] \neq 3$. We first show that then $[G: H]=2$. If this is not the case then $[G: H] \geqslant 4$. Consider now any $a \in S_{3}$ and note that since $\Gamma$ is bipartite and $t a^{-1} \notin S$ we have that $\partial\left(t, a^{-1}\right)=3$. By Proposition 16 we have that $c_{3}=2$, and so there must exist a path $P$ of length 3 from $t$ to $a^{-1}$, different from $\left(t, 1, t a, a^{-1}\right)$. By Lemma 13 this path must be of the form $\left(t, b, t b^{-1}, a^{-1}\right)$ for some $b \in S_{3}$. But as $[G: H] \geqslant 4$, Lemma 13 implies that $t b^{-1}$ and $a^{-1}$ do not belong to the same left coset of $H$, and consequently $t b^{-1} t=a^{-1}$, that is $b=a^{-1}$, which contradicts $a, b \in S_{3}$.

We can thus assume that $[G: H]=2$. Pick $a \in S_{3}$ and note that $t a \in H$ implies $a^{-1}=t a t \in t H$. As above, $\partial\left(t, a^{-1}\right)=3$. Since $c_{2}=1$, there are $c_{3}$ paths of length 3 from $t$ to $a^{-1}$. One of these paths is $\left(t, 1, t a, a^{-1}\right)$ and a similar argument as in the proof of Proposition 16 shows that all of the remaining ones are contained in $t H$. They are thus of the form $\left(t, b, t b^{-1} b^{\prime}, b b^{-1} b^{\prime \prime}\right)$ for some $b, b^{\prime}, b^{\prime \prime} \in S_{3}$ with $b b^{-1} b^{\prime \prime}=a^{-1}$.

If $b^{\prime}=a$, then $b^{\prime \prime}=b^{-1}$, contradicting the fact that $b \in S_{3}$. Therefore, $b^{\prime} \neq a$. By Proposition 16 it follows that for each $b^{\prime} \in S_{3} \backslash\{a\}$ there are precisely $c_{3}-2$ pairs $\left(b, b^{\prime \prime}\right) \in S_{3} \times S_{3}$ such that $b b^{\prime \prime}=a^{-1} b^{\prime}$, showing that there are $\left(\left|S_{3}\right|-1\right)\left(c_{3}-2\right)+1$ paths of length 3 from $t$ to $a^{-1}$. Therefore,

$$
c_{3}-1=\left(\left|S_{3}\right|-1\right)\left(c_{3}-2\right) .
$$

Since $\left|S_{3}\right|=|S|-1 \geqslant 2$, we thus have that $c_{3} \geqslant 3$, and so

$$
b_{0}-2=\left|S_{3}\right|-1=\frac{c_{3}-1}{c_{3}-2}=1+\frac{1}{c_{3}-2} .
$$

Since $b_{0}$ is an integer, this shows that $c_{3}=3$ and $b_{0}=4$. By [4], the intersection array of $\Gamma$ is $\{4,3,3,1 ; 1,1,3,4\}$, which is uniquely realized by the incidence graph of the Desarguesian affine plane of order 4 minus a parallel class of lines. However, one can verify (using a computer) that this graph has no presentation as a Cayley graph of a generalized dihedral group with respect to a minimal connection set. Therefore, $[G: H]=3$, as claimed.

Remark 18. We would like to point out that the incidence graph of the Desarguesian affine plane of order 4 minus a parallel class of lines from the above proof is indeed a Cayley graph (see for instance [5, Proposition 3.2]). In fact, it is a Cayley graph of the generalized dihedral group $G D\left(\mathbb{Z}_{4} \times \mathbb{Z}_{4}\right)$. But as already mentioned there exists no corresponding minimal connection set for it.

Proposition 19. With reference to Notation 12, assume that $c_{2}=1$. Then $A$ is an elementary abelian 3-group of rank at least 2 , that is $A \cong \mathbb{Z}_{3}^{\ell}$ for some $\ell \geqslant 2$.

Proof. By Proposition 17 we have that $[G: H]=3$. By Proposition 14 we have that $S_{1}=\varnothing, S_{2}=\{1\}$, and so Lemma 13 implies that $V(\Gamma)=H \cup t H \cup a^{-1} H$, for any $a \in S_{3}$. Moreover, $\Gamma$ is bipartite. By Proposition 17 we also have $c_{3}=2$. Pick $a \in S_{3}$ and observe 
that $\partial\left(t, a^{-1}\right)=3$. Namely, $\left(t, 1, t a, a^{-1}\right)$ is a path of length 3 between $t$ and $a^{-1}$, while $t$ and $a^{-1}$ are not adjacent as $a \in S_{3}$.

As $c_{3}=2$, the vertex $t$ has exactly two neighbours, which are at distance 2 from $a^{-1}$. One of them is of course 1 . Therefore, there exist a unique $b \in S_{3}$, such that $b$ is a neighbour of $t$ and is at distance 2 from $a^{-1}$. But this is only possible if the only neighbour of $b$, which is not contained in $t H$ (namely $b t=t b^{-1}$ ), is contained in $a^{-1} H$ and is adjacent with $a^{-1}$. Therefore, $a^{-1} b^{-1} \in S_{3}$. But $a^{-1} b^{-1}$ is also a neighbour of $t$, which is at distance 2 from $a^{-1}$ (their common neighbour is $t a b$ ), and so we have that $b=a^{-1} b^{-1}$. Since $a$ is also the unique neighbour of $t$ which is at distance 3 from $b^{-1}$, repeating the above argument yields $a=b^{-1} a^{-1}=b$. It follows that $a^{3}=1$. Since this holds for any $a \in S_{3}$ and $S=\{t\} \cup\left\{t a \mid a \in S_{3}\right\}$ it thus clearly follows that all nontrivial elements of $A$ are of order 3 . Therefore, $A \cong \mathbb{Z}_{3}^{\ell}$ for some $\ell \geqslant 1$. In fact, $\ell \geqslant 2$, as otherwise $|H|=2$, in which case $\Gamma$ is the cycle of length 6 , which contradicts our assumption.

Remark 20. In what follows $A$ will always be isomorphic to the group $\mathbb{Z}_{3}^{\ell}$ for some $\ell \geqslant 2$. Note that we can view $\mathbb{Z}_{3}^{\ell}$ also as a vector space over $\mathbb{Z}_{3}$. When we take this viewpoint we will use some vector space terminology for the elements of $A$ (we will for example say that $a, b \in A$ are linearly independent). Observe that in order for $\Gamma$ to be connected, $S_{3}$ must contain $\ell$ linearly independent elements of $A$.

Proposition 21. With reference to Notation 12, assume that $c_{2}=1$. If $|A|=9$, then $\Gamma$ is isomorphic to the Pappus graph.

Proof. As $|A|=9$, the graph $\Gamma$ has 18 vertices. Recall also that, by Proposition 17, we have $[G: H]=3$. As $\Gamma$ is a bipartite regular graph with valency $k$ and intersection number $c_{2}=1$, we have that $1+k+k(k-1) \leqslant 18$, and so $k \in\{3,4\}$. By [4] there are no distance-regular graphs with valency 4 on 18 vertices. So $k=3$, which forces $S_{3}=\{a, b\}$ for some linearly independent $a, b \in A$. It is easy to verify that $\Gamma$ is isomorphic to the Pappus graph in this case.

Lemma 22. With reference to Notation 12, assume that $c_{2}=1$ and $A \cong \mathbb{Z}_{3}^{\ell}$ for some $\ell \geqslant 3$. Then the following (i)-(iii) hold:

(i) For any linearly independent elements $a, b \in S_{3}$ none of $a b, a^{2} b, a b^{2}, a^{2} b^{2}$ is contained in $S_{3}$.

(ii) For any triple of distinct elements $a, b, c \in S_{3}, a b^{2} c \notin S_{3}$.

(iii) $c_{4} \geqslant 3$.

Proof. By Proposition 17, we have that $[G: H]=3$. Pick linearly independent $a, b \in$ $S_{3}$. If $a b \in S_{3}$, then $t=$ tatabtb $\in H$, a contradiction. Similarly, if $a b^{2} \in S_{3}$, then $t=t a b^{2} t a t b \in H$ (recall that $b$ is of order 3), a contradiction. Exchanging the roles of $a$ and $b$ shows that $b a^{2} \notin S_{3}$. Finally, if $a^{2} b^{2} \in S_{3}$, then $t a t a^{2} b^{2}=a b^{2}=t b t a$, contradicting $c_{2}=1$. This proves (i). 
Let $a, b, c \in S_{3}$ be pairwise distinct. As $t a b^{2} c=$ tatbtc, $c_{2}=1$ implies that $a b^{2} c \in S_{3}$ can only occur if $t a=t a b^{2} c$, that is $c=b^{-1}$, contradicting the fact that $b, c \in S_{3}$. This proves (ii).

To prove (iii) let $a, b, c \in S_{3}$ be linearly independent (recall that $\ell \geqslant 3$ ) and consider the vertex $a b c$. For any of the 6 choices for $x, y, z$, where $\{x, y, z\}=\{a, b, c\}$, we get a walk of length 4 from 1 to $a b c$ by taking $\left(1, t x, x^{2} y, t x^{2} y^{2}, x y z\right)$. Since $c_{2}=1$ and $c_{3}=2$ (by Proposition 16) it thus suffices to show that $\partial(1, a b c)=4$. By (i) above $\partial\left(1, t a^{2} b^{2}\right)=3$. Since $\left(1, t a, a^{2} b, t a^{2} b^{2}\right)$ and $\left(1, t b, a b^{2}, t a^{2} b^{2}\right)$ are paths of length 3 from 1 to $t a^{2} b^{2}, c_{2}=1$ and $c_{3}=2$ imply that any other neighbour of $t a^{2} b^{2}$ is at distance 4 from 1. As $a b c=t a^{2} b^{2} t c$ is such a neighbour (recall that $a, b, c$ are linearly independent), this shows that $\partial(1, a b c)=4$, as claimed.

Proposition 23. With reference to Notation 12, assume that $c_{2}=1$. Then $\Gamma$ is isomorphic to the Pappus graph.

Proof. By Propositions 14, 16, 17 and 19 we have that $S_{1}=\varnothing, S_{2}=\{1\}$, the graph $\Gamma$ is bipartite, $[G: H]=3, c_{3}=2$ and $A \cong \mathbb{Z}_{3}^{\ell}$ for some $\ell \geqslant 2$. By Proposition 21 it thus suffices to show that $\ell=2$.

By way of contradiction assume that $\ell \geqslant 3$ and recall that in this case Lemma 22 implies that $c_{4} \geqslant 3$. Pick linearly independent $a, b, c \in S_{3}$ and consider the vertex $a b^{2} c=$ ttatbtc $\in A \cap t H$ (recall that $t a, t b, t c \in H$ ). Since $[G: H]=3$, Lemma 13 implies that $a b^{2} c t \notin H$, while Lemma 22 implies that $a b^{2} c \notin S_{3}$, and so $\partial\left(1, a b^{2} c\right) \geqslant 4$. Since

$$
\left(1, t, a, t a^{2} b, a b^{2} c\right) \text { and }\left(1, t, c, t b c^{2}, a b^{2} c\right)
$$

are two paths of length 4 from 1 to $a b^{2} c$, this shows that $\partial\left(1, a b^{2} c\right)=4$. As $c_{3}=2$ and $c_{4} \geqslant 3$, there thus exists a neighbour $t x$ of $a b^{2} c$, different from $t a^{2} b$ and $t b c^{2}$, such that $\partial(1, t x)=3$.

Recall that, since $[G: H]=3$, Lemma 13 implies that $G=H \cup t H \cup a^{-1} H$. We first show that $t x \in a^{-1} H$. If this is not the case, then $t x \in t H$, and so the fact that $c_{3}=2$ implies that $t x$ has two neighbours which are at distance 2 from 1 . By Lemma 13 at least one of them is contained in $t H$, let us call it $y$. By Lemma 13 the only neighbour of $y$ outside $t H$ is in $a^{-1} H$, and so cannot be adjacent to 1 , and so $y$ must be adjacent to $t$. But as $t x, t a^{2} b$ and $t c^{2} b$ are now three distinct vertices in $N\left(a b^{2} c\right) \cap N_{2}(t)$ and $\partial\left(t, a b^{2} c\right)=3$, this contradicts $c_{3}=2$.

We are thus left with the possibility that $t x \in a^{-1} H$, in which case Lemma 13 implies that $t x=a b^{2} c t=t a^{2} b c^{2}$, that is $x=a^{2} b c^{2}$. By Lemma 13 any path of length 3 from $t x$ to 1 must be of the form $\left(t x, x^{2} y, t x y^{2}, 1\right)$ for some $y \in S_{3}$ such that $x y^{2} \in S_{3}$ as well. There thus exists $y \in S_{3}$ such that $x y^{2}=a^{2} b c^{2} y^{2} \in S_{3}$. Note that, by Lemma $22, y \notin\{a, b, c\}$. But now there are at least three different paths of length 3 from 1 to $t a^{2} c y$ (namely $\left(1, t b, a^{2} c^{2} y^{2}, t a^{2} c y\right),\left(1, t c, a c^{2}, t a^{2} c y\right)$ and $\left.\left(1, t y, a y^{2}, t a^{2} c y\right)\right)$, so that $c_{3}=2$ and the fact that $\Gamma$ is bipartite imply that $t a^{2} c y$ is adjacent to 1 . But then $a^{2} c y \in S_{3}$, contradicting Lemma 22. This finally proves that $\ell=2$, as claimed.

Combining together the remarks from Section 3 and the results of Sections 5 and 6 we finally get a proof of Theorem 1 . 


\section{References}

[1] A. Abdollahi, E. R. van Dam and M. Jazaeri, Distance-regular Cayley graphs with least eigenvalue -2, Des. Codes Cryptogr., 84 (2017), 73-85.

[2] W. G. Bridges and R. A. Mena, Rational circulants with rational spectra and cyclic strongly regular graphs, Ars Combin., 8 (1979), 143-161.

[3] A. E. Brouwer, A. M. Cohen and A. Neumaier, Distance-regular graphs, SpringerVerlag, New York (1998).

[4] A. E. Brouwer and J. H. Koolen, The distance-regular graphs of valency four, J. Algebraic Combin., 10 (1999), 5-24.

[5] E. R. van Dam and M. Jazaeri, Distance-regular Cayley graphs with small valency, Ars Math. Contemp., 17 (2019), 203-222.

[6] Y. I. Leifman and M. Muzychuk, Strongly regular Cayley graphs over the group $\mathbb{Z}_{p^{n}} \oplus \mathbb{Z}_{p^{n}}$, Discrete Math., 305 (2005), 219-239.

[7] S. L. Ma, A Survey of Partial Difference Sets, Des. Codes Cryptogr., 4 (1994), 221261.

[8] S. L. Ma, Partial Difference Sets, Discrete Math., 52 (1984), 75-89.

[9] D. Marušič, Strong regularity and circulant graphs, Discrete Math., 78 (1989), 119 125.

[10] Š. Miklavič and P. Potočnik, Distance-regular circulants, Europ. J. Combin. 24 (2003), 777-784.

[11] Š. Miklavič and P. Potočnik, Distance-regular Cayley graphs on dihedral groups, J. Combin. Theory Ser. B, 97 (2007), 14-33.

[12] Š. Miklavič and P. Šparl, On distance-regular cayley graphs on abelian groups, J. Combin. Theory Ser. B, 108 (2014), 102-122.

[13] S. S. Shrikhande, The uniqueness of the $L_{2}$ association scheme, Ann. of Math. Stat., 30 (1959), 781-798.

[14] D. Stevanovć, Distance Regularity of Compositions of Graphs, Appl. Math. Lett., 17 (2004), 337-343. 